Research Paper

\title{
Prevalence and Genotype Distribution of HPV Infection in China: Analysis of 51,345 HPV Genotyping Results from China's Largest CAP Certified Laboratory
}

\author{
Zhengyu Zeng ${ }^{* 1}$, Huaitao Yang*2, Zaibo Li³ ${ }^{3}$ Xuekui He1, Christopher C. Griffith", Xiamen Chen ${ }^{1}$, Xiaolei \\ $\mathrm{Guo}^{1}$, Baowen Zheng ${ }^{1}$, Shangwei $\mathrm{Wu}^{1}$, Chengquan Zhao ${ }^{\mathrm{\varpi}}$ \\ 1. Guangzhou Kingmed Diagnostics, Guangzhou, Guangdong 510330, P. R. China. \\ 2. Department of Pathology, University Cincinnati College of Medicine, Cincinnati, OH 45219, USA \\ 3. Department of Pathology, Wexner Medical Center at Ohio State University, Columbus, OH 43210, USA. \\ 4. Department of Pathology, Emory University, Atlanta, GA, 30342, USA. \\ 5. Department of Pathology, Magee-Womens Hospital, University of Pittsburgh Medical Center, Pittsburgh, PA 15213, USA. \\ * Both authors made equal contribution for this paper. \\ $\triangle$ Corresponding author: Chengquan Zhao, MD, Department of Pathology, Magee-Womens Hospital, University of Pittsburgh Medical Center, Pittsburgh, PA \\ 15213, USA. Tel:+412-641-6678, Fax:+412-641-1675, Email zhaoc@upmc.edu
}

(C) Ivyspring International Publisher. Reproduction is permitted for personal, noncommercial use, provided that the article is in whole, unmodified, and properly cited. See http://ivyspring.com/terms for terms and conditions.

Received: 2016.01.14; Accepted: 2016.04.28; Published: 2016.05.25

\begin{abstract}
Introduction: The prevalence of cervical Human Papillomavirus (HPV) infection varies greatly worldwide and data regarding HPV prevalence and genotypes in China are limited.

Methods: HPV testing results were retrospectively examined at KingMed Diagnostics, the largest independent pathology laboratory in China, from January 2011 to June 2014. All testing was performed using the 26 HPV Genotyping Panel of Tellgenplex TM xMAPTM HPV DNA Test assay (TELLGEN, Shanghai, China). Overall prevalence, age-specific prevalence and genotype distributions were analyzed.

Results: A total of 51,345 samples were tested and the overall HPV prevalence was $26 \%$, with $21.12 \%$ positive for high risk (HR) HPV and $8.37 \%$ positive for low risk HPV. $80 \%$ of HPV positive cases were positive for a single HPV type. The three most common HR HPV types detected were HPV-52, -16, and -58 , in descending order. HPV-18 was only the $6^{\text {th }}$ most common type. When women were divided into three age groups: $<30,30-49, \geq 50$ years, HR HPV had the highest prevalence rate in women $<30$ years, and the lowest rate in women 30-49 years of age. The distribution of HR HPV genotypes also varied among these three age groups.

Conclusions: To the best of our knowledge, this is largest routine clinical practice report of HPV prevalence and genotypes in a population of women having limited cervical cancer screening. HPV-52 was the most prevalent HR HPV type in this population of women followed by HPV-16 and HPV-58. The overall and age-specific prevalence and genotype distribution of HR HPV are different in this Chinese population compared to that reported from Western countries.
\end{abstract}

Key words: cervical cancer, HPV, genotyping, prevalence, China

\section{Introduction}

Cervical cancer remains an important public health problem in Chinese women, especially among women living in rural China. ${ }^{1}$ Most cervical cancer and its precursors are caused by persistent high-risk Human Papillomavirus (HPV) infection. ${ }^{2}$ More than $200 \mathrm{HPV}$ genotypes have been characterized to date. Of these, 25 genotypes are classified based on their carcinogenicity in humans ${ }^{3}$. Detection and genotyping of HPV has been an essential tool for screening, diagnosis, and management of HPV-related cervical cancer and its precursor lesions.

The distribution of HPV genotypes in cervical cancer varies geographyically.3,4 A meta-analysis of 48 studies showed that HPV-16 and HPV-18 are the most 
prevalent genotypes worldwide. ${ }^{5}$ Based on WHO worldwide data, the five most frequent HR HPV types in patients with cervical cancer are HPV-16, -18, $-33,-45$ and -31 , while the most common types in the general female population are HPV-16,-18,-31,-58 and $-52 .{ }^{4}$ In contrast, $\mathrm{WHO}$ data indicate that the five most frequent HPV types in Chinese women with cervical cancer are HPV-16, -18, -58, -33 and -52 while HPV-16, $-52,-18,-51$ and -58 are the most common in the general population of Chinese women. ${ }^{4}$ Data on HPV genotype distribution and prevalence based on the Bethesda System (TBS) for Reporting Cervical Cytology are limited in China but most studies indicate that HPV-16 is most common; however, these studies also suggest that HPV-18 is not as common and indicate HPV-52 and -58 to be more prevalent than HPV-18 in the general population and cancer patients in China. ${ }^{6-9}$

We recently published a five-year experience (2007-2012) on the cytology reporting profile of China's largest independent laboratory, KingMed Laboratory, which has achieved accreditation through the College of American Pathologists (CAP) international Laboratory Accreditation Program (LAP) and demonstrated that Bethesda System cytology reporting rates within a database of almost 1,400,000 Papanicolaou (Pap) test reports were largely within established CAP benchmark ranges. ${ }^{10,11}$ Another study indicated HR HPV positive rates of more than $20 \%$ in all age groups from over 500,000 tested Chinese women in the same laboratory. ${ }^{12}$ In this extended serial study, a retrospective database search from January 2011 to June 2014 was conducted from KingMed Diagnostics to analyze HPV testing results using a 26 HPV Genotyping Panel of Tellgenplex ${ }^{\mathrm{TM}} \times \mathrm{xMAP}^{\mathrm{TM}}$ HPV DNA Test assay (TELLGEN, Shanghai, China). Overall prevalence, age-specific prevalence and genotype distributions were analyzed.

\section{Materials and Method}

This retrospective study was designed to document all cervical HPV test results reported at Guangzhou Kingmed Diagnostics of Guangzhou, China from January 2011 to June 2014. Guangzhou Kingmed Diagnostics is the largest independent laboratory in China and all HPV testing is performed in the CAP accredited Microbiology laboratory. Cervical samples for HPV genotyping were collected from 364 hospitals, clinics, and physical examination centers in Guangdong (55\%) and other provinces (45\%). The decision to order HPV genotype testing was made by clinicians and patients on an individual basis as there are no standard guidelines in China. The documented reason for HPV testing varied and in the majority of cases was not stated. In addition to HPV testing some patients also had Pap cytology performed at the same time; however, most Pap cytology testing was performed at local hospitals and these results were not always available.

Samples were stored in the standard media provided with the kit. The presence and genotype of HPV was analyzed using the 26 HPV Genotyping Panel kit from Tellgen $p l e x^{\mathrm{TM}} \times \mathrm{xMAP}^{\mathrm{TM}}$ (TELLGEN Life Science Co. Ltd., Shanghai, China). This multiplex PCR technique detects 18 IARC Monograph high risk (HR) HPV genotypes $(-16,-18,-26,-31,-33,-35,-39,-45,-51$, $-52,-53,-56,-58,-59,-66,-68,-73$, and -82$)$, and 8 IARC Monograph low risk (LR) HPV genotypes (-6, -11, -61, $-40,-42,-44,-55$, and -83).3,13,14 Additionally, a Luminex's xMAP-based assay combines flow cytometry with color-coded microspheres ${ }^{14,15}$, which performs discrete assays on the surface of color coded beads/microspheres that are read by a Luminex analyzer. Briefly, each color-coded bead set is coated with a pre-designed HPV type-specific probe. The HPV DNA that is present in the sample is amplified via PCR using biotin-labeled consensus PCR primers. Amplified PCR products are hybridized to sets of beads with coated HPV type-specific probes. After subsequent incubation with phycoerythrin (PE)-conjugated streptavidin (SA-PE), beads are read within a Luminex 200 system (Luminex Corporation, Texas). Based on the unique fluorescent dye signature that is carried by each set of beads the HPV genotype can be determined unambiguisly. ${ }^{14-16}$

\section{Results}

\section{Overall HPV Prevalence}

The overall prevalence of detectable HPV infection was $26.0 \%(13,349 / 51,345)$ during the study period of January 2011 to June 2014. The prevalence of HR HPV types was $21.12 \%(10,844 / 51,345)$ and $8.37 \%$ $(4,298 / 51,345)$ for LR HPV types.

When women were divided into three age groups of $<30$ years, $30-49$ years, and $\geq 50$ years $(2,181$ women were excluded due to unknown age), the highest overall prevalence of HPV was found in women $<30$ years old $(4991 / 15772,31.65 \%)$ while the lowest HPV prevalence was found in women 30-49 years old $(6552 / 28998,22.60 \%)$. Overall HPV prevalence in women $30-49$ years was $26.97 \%(1185 / 4394)$.

The rate of HR HPV types also differed among these age groups. Similar to the overall prevalence of $\mathrm{HPV}$, women $<30$ years of age also had the highest any HR HPV (33.83\%) and the lowest any HR HPV types was found in women 30-49 years of age (22.79\%). Meanwhile, the age-specific any LR HPV positive rate was $14.49 \%, 5.88 \%$ and $8.31 \%$ in these age groups ( $<30$ years, $30-49$ years, and $\geq 50$ years, respectively) (Table 1 ). 
Table 1. Distribution of Any IARC HR and LR HPV Types by Age groups

\begin{tabular}{llllll}
\hline HPV & $<30 y$ & $30-49 y$ & $\geq 50 y$ & Unknown & All ages \\
Types & $\mathrm{N}=15772$ & $\mathrm{~N}=28998$ & $\mathrm{~N}=4394$ & $\mathrm{~N}=2181$ & $\mathrm{~N}=51345$ \\
\hline HR & 5335 & 6608 & 1289 & 634 & 13866 \\
HPV & $(33.83 \%)$ & $(22.79 \%)$ & $(29.34 \%)$ & $(29.07 \%)$ & $(27.01 \%)$ \\
LR & 2285 & 1705 & 365 & 237 & 4682 \\
HPV & $(14.49 \%)$ & $(5.88 \%)$ & $(8.31 \%)$ & $(10.87 \%)$ & $(9.12 \%)$ \\
\hline
\end{tabular}

The percentages were calculated in the formula of "positive HPV tests/all 51345 tested women"

Abbreviations - IARC: International Agency for Research on Cancer; HPV; human papillomavirus; HR HPV: high risk HPV; LR HPV: low risk HPV; N: number; y: years.

\section{HR HPV Genotype distribution}

In all tested women, the most commonly detected HR HPV genotype was HPV-52 (5.05\%). The next most common HR HPV genotypes were HPV-16 (4.76\%) and HPV-58 (2.93\%). HR HPV genotypes with a prevalence of more than $1 \%$ included HPV-56, -39 , $-18,-59,-68,-33$, and -51 (table 2). Additionally, when looking at the rates of each of these genotypes by age groups ( $<30$ years, $30-49$ years, and $\geq 50$ years), HR HPV genotype distributions were also different (Table 2). Based on prevalence, HPV-52 was most common in women $<30$ years of age $(5.84 \%)$ and women $30-49$ years of age $(4.47 \%)$. However, older women $\geq 50$ years of age, were most commonly positive for HPV-16 (5.92\%), with HPV-52 being the second most common genotype detected $(5.87 \%)$.

The six most common HR HPV genotypes in younger women $<30$ years of age were HPV-52 $(5.84 \%),-16(5.34 \%),-58$ (3.18\%), -59 (2.48\%), -39 $(2.39 \%)$, and $-56(2.36 \%)$. The six most common HR HPV genotypes in women of 30-49 years of age were HPV-52 (4.47\%), -16 (4.29\%), -58 (2.69\%), -18 (1.33\%), $-39(1.32 \%)$ and $-56(1.28 \%)$. These two age groups (<30 years and 30-49 years) had the same top three most common HR HPV types: HPV-52, -16 and -58 . While the same three HPV genotypes were also most common in older women $\geq 50$ year, HPV-16 (5.92\%) was slightly more common than HPV-52 (5.87\%) in this older population. The next most common HPV types in older women $\geq 50$ year in decreasing order were HPV-58 (3.32\%), -56 (2.14\%), -18 (1.57\%), and -68 (1.46\%). In all age groups, HR HPV-35, $-45,-26$ and -73 were consistently the four least frequently detected HR HPV genotypes (prevalence ranged from $0.01 \%-0.60 \%$ ) (Table 2).

Table 2: Prevalence and Distribution for Any HPV Type for Tested 51345 Women

\begin{tabular}{|c|c|c|c|c|c|c|c|c|c|c|c|c|c|c|c|}
\hline & \multirow{2}{*}{\multicolumn{3}{|c|}{$\begin{array}{l}\text { Overall Prevalence } \\
\text { All ages }\end{array}$}} & \multicolumn{12}{|c|}{ Prevalence by age group } \\
\hline & & & & \multicolumn{2}{|l|}{$<30 y$} & \multicolumn{3}{|c|}{30 to $49 y$} & \multicolumn{3}{|c|}{$>=50 \mathrm{y}$} & \multicolumn{3}{|c|}{ unknown } & \\
\hline & \multicolumn{3}{|c|}{ Overall total cases $n=51345$} & \multicolumn{2}{|c|}{$\mathrm{n}=15772$} & \multicolumn{3}{|c|}{$n=28998$} & \multicolumn{3}{|c|}{$n=4394$} & \multicolumn{3}{|c|}{$\mathrm{n}=\mathbf{2 1 8 1}$} & \\
\hline & $\begin{array}{l}\text { Gen- } \\
\text { otype }\end{array}$ & $\begin{array}{l}\text { No. Posi- } \\
\text { tive cases }\end{array}$ & $\begin{array}{l}\text { Preva- } \\
\text { lence }(\%)\end{array}$ & $\begin{array}{l}\text { Gen- } \\
\text { otypes }\end{array}$ & $\begin{array}{l}\text { No. posi- } \\
\text { tive cases }\end{array}$ & $\begin{array}{l}\text { Preva- } \\
\text { lence }(\%)\end{array}$ & $\begin{array}{l}\text { Gen- } \\
\text { otypes }\end{array}$ & $\begin{array}{l}\text { No. posi- } \\
\text { tive cases }\end{array}$ & $\begin{array}{l}\text { Preva- } \\
\text { lence }(\%)\end{array}$ & $\begin{array}{l}\begin{array}{l}\text { Gen- } \\
\text { otypes }\end{array} \\
\end{array}$ & $\begin{array}{l}\text { No. posi- } \\
\text { tive cases }\end{array}$ & $\begin{array}{l}\text { Preva- } \\
\text { lence }(\%)\end{array}$ & $\begin{array}{l}\text { Gen- } \\
\text { otypes }\end{array}$ & $\begin{array}{l}\text { No. posi- } \\
\text { tive cases }\end{array}$ & $\begin{array}{l}\text { Preva- } \\
\text { lence (\%) }\end{array}$ \\
\hline \multirow{18}{*}{$\begin{array}{l}\text { IARC HR } \\
\text { HPV types }\end{array}$} & 52 & 2595 & 5.05 & 52 & 921 & $5.84 \%$ & 52 & 1295 & $4.47 \%$ & 16 & 260 & $5.92 \%$ & 52 & 121 & $5.50 \%$ \\
\hline & 16 & 2446 & 4.76 & 16 & 842 & $5.34 \%$ & 16 & 1243 & $4.29 \%$ & 52 & 258 & $5.87 \%$ & 16 & 101 & $4.60 \%$ \\
\hline & 58 & 1504 & 2.93 & 58 & 502 & $3.18 \%$ & 58 & 781 & $2.69 \%$ & 58 & 146 & $3.32 \%$ & 58 & 75 & $3.40 \%$ \\
\hline & 56 & 877 & 1.71 & 59 & 391 & $2.48 \%$ & 18 & 385 & $1.33 \%$ & 56 & 94 & $2.14 \%$ & 56 & 40 & $1.80 \%$ \\
\hline & 39 & 858 & 1.67 & 39 & 377 & $2.39 \%$ & 39 & 384 & $1.32 \%$ & 18 & 69 & $1.57 \%$ & 18 & 37 & $1.70 \%$ \\
\hline & 18 & 801 & 1.56 & 56 & 372 & $2.36 \%$ & 56 & 371 & $1.28 \%$ & 68 & 64 & $1.46 \%$ & 33 & 36 & $1.70 \%$ \\
\hline & 59 & 779 & 1.52 & 18 & 310 & $1.97 \%$ & 68 & 347 & $1.20 \%$ & 39 & 61 & $1.39 \%$ & 39 & 36 & $1.70 \%$ \\
\hline & 68 & 719 & 1.4 & 51 & 289 & $1.83 \%$ & 33 & 306 & $1.06 \%$ & 59 & 57 & $1.30 \%$ & 68 & 33 & $1.50 \%$ \\
\hline & 33 & 631 & 1.23 & 68 & 275 & $1.74 \%$ & 59 & 300 & $1.03 \%$ & 33 & 55 & $1.25 \%$ & 59 & 31 & $1.40 \%$ \\
\hline & 51 & 613 & 1.19 & 33 & 234 & $1.48 \%$ & 51 & 267 & $0.92 \%$ & 53 & 48 & $1.09 \%$ & 51 & 23 & $1.10 \%$ \\
\hline & 31 & 454 & 0.88 & 66 & 188 & $1.19 \%$ & 31 & 252 & $0.87 \%$ & 31 & 38 & $0.86 \%$ & 82 & 20 & $0.90 \%$ \\
\hline & 66 & 410 & 0.8 & 82 & 177 & $1.12 \%$ & 66 & 174 & $0.60 \%$ & 51 & 34 & $0.77 \%$ & 53 & 17 & $0.80 \%$ \\
\hline & 82 & 385 & 0.75 & 31 & 147 & $0.93 \%$ & 53 & 169 & $0.58 \%$ & 66 & 31 & $0.71 \%$ & 66 & 17 & $0.80 \%$ \\
\hline & 53 & 338 & 0.66 & 53 & 104 & $0.66 \%$ & 82 & 158 & $0.54 \%$ & 82 & 30 & $0.68 \%$ & 31 & 17 & $0.80 \%$ \\
\hline & 35 & 213 & 0.41 & 35 & 94 & $0.60 \%$ & 35 & 82 & $0.28 \%$ & 35 & 21 & $0.48 \%$ & 35 & 16 & $0.70 \%$ \\
\hline & 45 & 198 & 0.39 & 45 & 86 & $0.55 \%$ & 45 & 82 & $0.28 \%$ & 45 & 19 & $0.43 \%$ & 45 & 11 & $0.50 \%$ \\
\hline & 26 & 29 & 0.06 & 26 & 16 & $0.10 \%$ & 26 & 9 & $0.03 \%$ & 26 & 2 & $0.05 \%$ & 26 & 2 & $0.10 \%$ \\
\hline & 73 & 16 & 0.03 & 73 & 10 & $0.06 \%$ & 73 & 3 & $0.01 \%$ & 73 & 2 & $0.05 \%$ & 73 & 1 & $0.80 \%$ \\
\hline $\begin{array}{l}\text { Subtotal-any } \\
\text { HPV type }\end{array}$ & & 13866 & & & 5335 & & & 6608 & & & 1289 & & & 634 & \\
\hline \multirow{8}{*}{$\begin{array}{l}\text { IARC LR } \\
\text { HPV Type }\end{array}$} & 6 & 1732 & 3.37 & 6 & 33 & $6.61 \%$ & 6 & 545 & $1.88 \%$ & 61 & 95 & $2.16 \%$ & 6 & 79 & $3.60 \%$ \\
\hline & 11 & 1084 & 2.11 & 11 & 16 & $4.24 \%$ & 61 & 310 & $1.07 \%$ & 6 & 66 & $1.50 \%$ & 11 & 57 & $2.60 \%$ \\
\hline & 61 & 685 & 1.33 & 61 & 1042 & $1.50 \%$ & 11 & 301 & $1.04 \%$ & 44 & 59 & $1.34 \%$ & 61 & 43 & $2.00 \%$ \\
\hline & 44 & 389 & 0.76 & 40 & 668 & $0.81 \%$ & 44 & 215 & $0.74 \%$ & 11 & 58 & $1.32 \%$ & 55 & 24 & $1.10 \%$ \\
\hline & 55 & 341 & 0.67 & 55 & 237 & $0.67 \%$ & 55 & 175 & $0.60 \%$ & 55 & 36 & $0.82 \%$ & 44 & 13 & $0.60 \%$ \\
\hline & 40 & 219 & 0.43 & 44 & 128 & $0.65 \%$ & 42 & 62 & $0.21 \%$ & 40 & 21 & $0.48 \%$ & 42 & 8 & $0.40 \%$ \\
\hline & 42 & 147 & 0.29 & 42 & 102 & $0.37 \%$ & 40 & 62 & $0.21 \%$ & 42 & 18 & $0.41 \%$ & 40 & 8 & $0.40 \%$ \\
\hline & 83 & 85 & 0.17 & 83 & 59 & $0.21 \%$ & 83 & 35 & $0.12 \%$ & 83 & 12 & $0.27 \%$ & 83 & 5 & $0.20 \%$ \\
\hline $\begin{array}{l}\text { Subtotal-any } \\
\text { HPV type }\end{array}$ & & 4682 & & & 2285 & & & 1705 & & & 365 & & & 237 & \\
\hline $\begin{array}{l}\text { Total positive } \\
\text { patients }\end{array}$ & & 13349 & 26 & & 4991 & 31.65 & & 6552 & 22.6 & & 1185 & 26.97 & & 621 & 28.47 \\
\hline
\end{tabular}




\section{LR HPV Genotype distribution}

In all tested women, the three most commonly detected LR HPV genotypes were HPV-6 (3.37\%), -11 $(2.11 \%)$ and $-61(1.33 \%)$ (Table 2$)$. In women less than 50 years of age (i.e. age groups $<30$ years and $30-49$ years), HPV-6 was the most commonly detected LR HPV genotype $(6.61 \%$ and $1.88 \%$, respectively) although the prevalence of HPV-6 in younger women $<30$ years was three times that seen in women of 30-49 years of age. In contrast, in older women $\geq 50$ years of age, HPV-61 was the most commonly detected LR HPV genotype (2.16\%).

\section{Distribution of Single and Multiple HR HPV infections}

When examining the possibility of co-infection by multiple HR HPV genotypes it was found that single HR HPV infections were most common in infected women with an overall prevalence of $18.8 \%$, accounting for $72.33 \%$ of HR HPV positive cases. The overall rate of multiple HR HPV type infection was $7.19 \%$, accounting for $27.67 \%$ of HR HPV positive cases. Of the women with multiple HPV infections, infection by two types (double infection) was most common with an overall prevalence of $5.10 \%$ (Table 3). Three HPV genotypes (triple infection) were found in 750 women for an overall prevalence of $1.55 \%$. Only $0.6 \%$ of women had four or more detectable HR HPV genotypes.

Table 3. Distribution of Single and Multiple HR HPV infections

\begin{tabular}{llll}
\hline HR HPV Infections & $\begin{array}{l}\text { HR HPV posi- } \\
\text { tive case }\end{array}$ & Percentage $(x / 13349)$ & $\begin{array}{l}\text { Prevalence } \\
(\mathrm{x} / 51345)\end{array}$ \\
\hline Single HPV type & 9656 & $72.34 \%$ & $18.80 \%$ \\
Two HPV types & 2632 & $19.72 \%$ & $5.10 \%$ \\
Three HPV types & 750 & $5.62 \%$ & $1.50 \%$ \\
$\geq$ Four HPV types & 311 & $2.30 \%$ & $0.60 \%$ \\
Total & 13349 & $100 \%$ & $26.00 \%$ \\
\hline
\end{tabular}

Abbreviations - HPV; human papillomavirus; HR HPV: high risk HPV.

\section{Distribution for HR HPV, LR HPV and mixed type (both HR and LR) Infection}

Cases with HR HPV types only, LR HPV types only, or mixed HR and LR HPV infections were analyzed (Table 4). HR HPV only infection accounted for $67.80 \%$ of all $13,349 \mathrm{HPV}$ positive women with an overall prevalence of $17.63 \%$. 1,755 women had two or more HR HPV types (no LR HPV infection), accounting for $13.15 \%$ of all $13,349 \mathrm{HPV}$ positive cases. $4.88 \%$ women had LR HPV only infection. $13.43 \%$ of HPV positive cases showed both HR and LR HPV infections for an overall prevalence of $3.49 \%$ for all tested women.
Table 4. Distribution for HR and LR HPVs: Single Types and Mixed Types Infections of the 51345 Tested Women

\begin{tabular}{|c|c|c|c|c|c|c|c|}
\hline \multirow[t]{2}{*}{ HPV Types } & \multicolumn{2}{|c|}{ Single infection } & \multicolumn{2}{|c|}{$\begin{array}{l}\text { Multiple infec- } \\
\text { tions* }\end{array}$} & \multicolumn{3}{|c|}{ Total Cases } \\
\hline & Positive & $\%$ & Positive & $\%$ & Positive & $\%$ & Prevalence \\
\hline $\begin{array}{l}\text { HR HPV } \\
\text { types only }\end{array}$ & 7296 & 54.66 & 1755 & 13.15 & 9051 & 67.80 & $17.63 \%$ \\
\hline $\begin{array}{l}\text { LR HPV } \\
\text { types only }\end{array}$ & 2360 & 17.68 & 145 & 1.09 & 2505 & 18.77 & $4.88 \%$ \\
\hline $\begin{array}{l}\text { Mixed HR } \\
\text { and LR } \\
\text { HPV types }\end{array}$ & $1094^{* *}$ & 8.20 & 699 & 5.24 & 1793 & 13.43 & $3.49 \%$ \\
\hline Total & 10750 & 80.53 & 2599 & 19.47 & 13349 & 100 & $26.0 \%$ \\
\hline
\end{tabular}

*indicating two and more HR HPV types infections in HR HPV only group; two and more LR HPV types infections in LR HPV only group; Two and more HR HPV types or two and more LR HPV types infection in the mixed HR and LR HPV group

**indicating single HR HPV and single LR HPV mixed infection.

Abbreviations - HR HPV: high risk HPV; LR HPV: low risk HPV; \#: number; HPV; human papillomavirus.

\section{Discussion}

Studies of HPV prevalence and genotype distribution indicate that HPV-16 and -18 are the most prevalent viral types in cervical cancer and together are responsible for approximately $70 \%$ of cervical cancer cases globally. However, the prevalence and genotype distribution of HPV infection are known to vary substantially between different regions and populations. ${ }^{3-5}$ Studies have demonstrated that HPV prevalence and type distribution vary in women from different populations, different countries, different age groups and with different Pap cytology findings. Some studies have revealed a substantial difference in prevalence and genotype distribution of HR HPV infection among women from Western and Eastern countries. ${ }^{3-5,} 18-20$ The overall HPV prevalence in 157,879 women world-wide with normal cervical cytology was estimated to be $10.4 \%$ but when examined by region showed various rates of $22.1 \%$ in Africa, $20.4 \%$ in Central America and Mexico, $11.3 \%$ in Northern America, $8.1 \%$ in Europe, and $8.0 \%$ in Asia. The data on HPV prevalence and genotype distribution in the largely unscreened Chinese female population is limited.

Currently there is no well-established systematic national cancer registry or organized cervical cancer screening program in China and there is no uniform national standard for cervical cytology quality control. The current study, examining over 51,000 Chinese women, demonstrates an overall HPV prevalence of $26 \%$, with a HR HPV infection rate of $21.12 \%$ and a LR HPV infection rate of $8.37 \%$ in all age groups using the Tellgenplex ${ }^{\mathrm{TM}} 26$ HPV Genotyping Panel. This result is consistent with our recent report showing a $21.7 \%$ rate of HR HPV infection using Hybrid Capture 2 (HC2) in the same laboratory. ${ }^{12}$ Additionally, a similar population-based study of HPV geno- 
type prevalence in the United States, indicates a similar overall rate of HPV infection of $27.3 \%$ when 37 HPV types were tested. ${ }^{17}$

A Danish study described the prevalence of carcinogenics HPV genotypes from over 40,000 women in the general screening women $(94 \%$ with normal cytology and 6\% with abnormal cytology). ${ }^{21} \mathrm{HPV} 16$ was the significantly most prevalent type being present in $5.4 \%$ of all women and the most common type detected in CIN2, CIN3 and cervical cancers. ${ }^{21} \mathrm{~A}$ meta-analysis of 1 million women with normal cytology from 5 continents estimated global HPV prevalence was $11.7 \%$ with 5 most common types worldwide, with HPV16 at 3.2\% and HPV 18 at $1.4 \% .{ }^{22}$ The U. S. ATHENA (Addressing the Need for Advanced HPV Diagnostics) trial reported among 45,933 women 21 years or older undergoing routine cervical cancer screening, the prevalences of HR-HPV (14 genotypes), HPV 16, and HPV 18 were $12.5 \%, 2.8 \%$, and $1.0 \%$, respectively and HR-HPV and HPV 16 positivities were the most important risk factor for CINs. ${ }^{23}$ The overall prevalence of HR-HPV for women with negative cytology results was $9.8 \%$. ${ }^{23}$ Among the 32,260 women 30 years or older with negative cytology, the overall prevalence of HR-HPV detected using the cobas HPV Test was $6.7 \%$ and the overall prevalence rates for HPV16, HPV 18, and 12 other HR-HPV genotypes were $1.0 \%, 0.5 \%$, and $5.2 \%$, respectively. ${ }^{24}$

This is one of the largest studies to demonstrate HPV prevalence and genotype distribution in Chinese women. Our results from 51,345 Chinese women without routine cervical cancer screening, tested in China's largest CAP certified laboratory revealed HPV-52, rather than HPV-16, to be the most common high risk HPV genotype in the population of all tested Chinese women. When stratified by age, HPV-52 was most the most common HPV infection in women younger than 50 years of age, but HPV-16 was most common in women at least 50 years of age.

Several earlier studies from China have suggested that HPV-16 is the most common HR HPV type among all grades of cytological abnormalities ${ }^{6,18}$, consistent with results reported from Western countries. ${ }^{4}$ This same data also demonstrated HPV-52 and -58 to be quite common in Chinese women, and much more common than HPV-18 6, 18, different from the results seen in women from western countries. ${ }^{3-5,} 25$ Similar to our results, one study from the Guangdong province of China found HPV-52 to be the most prevalent HPV genotype in women without a correlation to Pap cytology results. ${ }^{26}$ Another study from Beijing in northern China found HPV-52 to be the most common HPV genotype in women with cervicitis or low grade dysplasia only 27 . Again however, HPV-16 was most common in women with high grade intraepithelial neoplasia or cervical cancer. A population-based study from Zhejiang Province of southeast China demonstrated similar findings with HPV-52 being most common in women younger than 50 years of age. ${ }^{28}$

The most likely explanation for the differences in these studies is the differences in the populations being studied. Our data as well as others examining HPV infection rates and genotype distributions that examined all tested women without correlation to Pap cytology results are likely to include many women with HPV infection but no detectable cervical abnormality/precursor lesion. When studies limit their results to only women with cervical abnormalities detected by cytology or correlate HPV test results with Pap cytology results, the literature indicates that HPV-16 is more common in women with detectable cervical abnormality. Unfortunately, cervical cytology results were not available to us for the women included in this study and therefore we are unable to correlate HPV infection and genotype rates with detectable cervical abnormalities. However, given the issues discussed above it can be hypothesised that while HPV-52 is the most common cause of HPV infection in Chinese women, it is less able to result in detectable cervical abnormality than HPV-16. These results are consistent with other smaller studies. ${ }^{26-28}$ Further study is needed to address the prevalence profile of HPV-52 in both cytology-normal and cytology-abnormal populations.

Here we demonstrate that single genotype HPV infections are most common, accounting for two third of all positive cases, consistent with previous smaller studies. ${ }^{8,29}$ Multiple HPV genotype infections are less common and some have suggested that such co-infections could potentially have competitive and/or cooperative interactions between HPV genotypes. The mechanisms and potential oncogenic effects of multiple genotype infections warrants further investigation and such data could be useful in the development of HPV prophylactic vaccines.

The limitation of this study is the fact that cervical cytology or histology results were not available for correlation with HPV genotype results. This was at least partly due to the fact that clinicians in China request Pap tests and/or HPV tests without the benefit of standardized guidelines. Some patients in our cohort had HPV testing performed by Guangzhou KingMed Diagnostics, while Pap cytology tests were performed in a separate hospital system and, therefore, not available for this study. Another limitation is sampling bias. Women in this study were not evenly distributed geographically. About half of the women in our cohort were from Guangdong province and the other half were from other provinces of China (45\%). 
The recently updated 2012 cervical cancer screening guideline in the United State clearly indicate that HPV testing should only look for high-risk genotypes; testing for low-risk HPV types unrelated to cervical cancer should not be used in cervical cancer screening. ${ }^{30}$ The 2013 ASCCP guideline indicate that genotyping for HPV-16 and -18 is an acceptable triage for women 30 years of age and older that are HR HPV-positive but cytology-negative after co-testing. When HPV-16 or -18 are detected, colposcopy is recommended. ${ }^{31}$ Given that HPV genotype distribution varies by region, it may be important to include additional HR HPV types in such triage for women from different regions. Further studies would need to be performed on a regional basis to determine if and which genotypes are most important in such scenarios.

To our knowledge, this is the largest study to analyze HPV genotyping results from daily clinical practice data instead of a population-based public health database. The data from this daily clinical practice regarding HPV results, especially HR HPV types, could be helpful for clinical management. This large retrospective study reports HPV prevalence and genotype distribution results in Chinese women where there is no well-established national cervical cancer screening program. Our data demonstrate that the three most prevalent HR HPV genotypes in descending order are HPV-52, -16 and -58 , in women younger than 50 years. The data provide valuable information for HPV based screening and prevention for women in China where robust screening and vaccination programs are not yet established. As the HPV types differ in the Chines population from those in the Western countries, for optimal population coverage, in addition to HPV 16 and 18, next generation HPV prophylactic vaccines including HPV-52 and -58 may offer higher protection for Chinese women. The unique epidemiological feature of HPV52 and 58 in China should be considered in the design and evaluation of diagnostic assays of cervical screening intended for Chinese women.

\section{Competing Interests}

The authors have declared that no competing interest exists.

\section{References}

[1] Shi JF, Qiao YL, Smith JS, et al. Epidemiology and prevention of human papillomavirus and cervical cancer in China and Mongolia. Vaccine 2008; 26 Suppl 12:M53-59.

[2] Walboomers JM, Jacobs MV, Manos MM, et al. Human papillomavirus is a necessary cause of invasive cervical cancer worldwide. J of pathol. 1999; 189:12-19.

[3] Human papillomaviruses. In: IARC, Humans WGotEoCRt: Biological agents. Volume $100 \mathrm{~B}$. A review of human carcinogens. IARC monographs on the evaluation of carcinogenic risks to humans / World Health Organization, International Agency for Research on Cancer 2012; 100:1-441.
[4] Castellsagué X, de Sanjosé S, Aguado KS, HPV WIICo, Cervical C: HPV and cervical cancer in the 2007 report. Vaccine 2007, 25 Suppl 3:C1-230.

[5] de Sanjose S, Diaz M, Castellsague X, et al. Worldwide prevalence and genotype distribution of cervical human papillomavirus DNA in women with normal cytology: a meta-analysis. The Lancet Infectious diseases. 2007; 7:453-459.

[6] Wu EQ, Liu B, Cui JF, et al. Prevalence of type-specific human papillomavirus and pap results in Chinese women: a multi-center, population-based cross-sectional study. Cancer causes \& control. CCC 2013; 24:795-803.

[7] Jing L, Zhong X, Huang W, et al. HPV genotypes and associated cervical cytological abnormalities in women from the Pearl River Delta region of Guangdong province, China: a cross-sectional study. BMC infectious diseases 2014, 14:388

[8] Wang L, Li J, Chen L. Prevalence of human papillomavirus and its genotype among 1336 invasive cervical cancer patients in Hunan province, central south China. Journal of medical virology. 2015; 87:516-521.

[9] Li J, Kang LN, Qiao YL. Review of the cervical cancer disease burden in mainland China. Asian Pacific journal of cancer prevention. APJCP 2011; 12:1149-1153.

[10] Zheng B, Austin RM, Liang X, et al. Bethesda system reporting rates for conventional papanicolaou tests and liquid-based cytology in a large chinese, college of american pathologists-certified independent medical laboratory: analysis of 1394389 papanicolaou test reports. Arch Path Lab Med. 2015; 139:373-377.

[11] Zheng B, Austin RM, Liang X, et al. Positive predictive value (PPV) of a high grade squamous intraepithelial lesion (HSIL) cervical cytology result in China's largest College of American Pathologists (CAP) certified laboratory. JASC 2015; (4):84-89.

[12] Zeng Z, , Austin RM, Xuekui H, et al. Prevalence of high risk human papillomavirus infection in China: analysis of 671,163 human papillomavirus test results from China's largest CAP-certified laboratory. Am J Clin Pathol. 2016 March 2 [Epub ahead of print]

[13] Doorbar J, Egawa N, Griffin H, et al. Human papillomavirus molecular biology and disease association. Rev Med Virol. 2015; 25 Suppl 1:2-23.

[14] Shen Y, Gong JM, Li YQ, et al. Epidemiology and genotype distribution of human papillomavirus (HPV) in women of Henan Province, China. Clin Chim Acta. 2013; 415:297-301.

[15] Cherne S, Popov V, Feng Q. Protocol for the detection and genotyping of human papillomaviruses using a liquid bead microarray assay. Methods Mol Biol. 2012; 903:205-23.

[16] Jiang HL, Zhu HH, Zhou LF, et al. Genotyping of human papillomavirus in cervical lesions by L1 consensus PCR and the Luminex xMAP system. J Med Microbiol. 2006; 55:715-20.

[17] Wheeler CM, Hunt WC, Cuzick J, et al. A population-based study of human papillomavirus genotype prevalence in the United States: baseline measures prior to mass human papillomavirus vaccination. Int J Cancer. 2013; 132(1):198-207.

[18] Jing L, Zhong X, Zhong Z, et al. Prevalence of human papillomavirus infection in guangdong province, china: a population-based survey of 78,355 women. SexTransmDis .2014; 41:732-738.

[19] Bruni L, Diaz M, Castellsague X, Ferrer E, Bosch FX, de Sanjose S. Cervical human papillomavirus prevalence in 5 continents: meta-analysis of 1 million women with normal cytological findings. J Infect Dis.2010; 202:1789-99.

[20] Li N, Franceschi S, Howell-Jones R, Snijders PJ, Clifford GM. Human papillomavirus type distribution in 30,848 invasive cervical cancers worldwide: Variation by geographical region, histological type and year of publication. Int J Cancer. 2011; 128:927-35.

[21] Kjær SK, Munk C, Junge J, Iftner T. Carcinogenic HPV prevalence and age-specific type distribution in 40,382 women with normal cervical cytology, ASCUS/LSIL, HSIL, or cervical cancer: what is the potential for prevention? Cancer Causes Control 2014 25(2):179-189

[22] Bruni L, Diaz M, Castellsagué X, Ferrer E, Bosch FX, de Sanjosé S. Cervical human papillomavirus prevalence in 5 continents: meta-analysis of 1 million women with normal cytological findings. J Infect Dis. 2010;202(12):1789-1799.

[23] Wright TC Jr, Stoler MH, Behrens CM, Sharma A, Sharma K, Apple R. Interlaboratory variation in the performance of liquid-based cytology: insights from the ATHENA trial. Int J Cancer. 2014;134(8):1835-1843.

[24] Wright TC Jr, Stoler MH, Sharma A. Evaluation of HPV-16 and HPV-18 genotyping for the triage of women with high-risk HPV+ cytology-negative results. Am J Clin Pathol. 2011;136(4):578-586

[25] Bosch FX, Burchell AN, Schiffman M, et al. Epidemiology and natural history of human papillomavirus infections and type-specific implications in cervical neoplasia. Vaccine. 2008; 26 Suppl 10:K1-16.

[26] Chen Q, Luo ZY, Lin M, et al. Prevalence and genotype distribution of human papillomavirus infections in women attending hospitals in Chaozhou of Guangdong province. APJCP 2012; 13:1519-24.

[27] Ding X, Liu Z, Su J, Yan D, Sun W, Zeng Z. Human papillomavirus type-specific prevalence in women referred for colposcopic examination in Beijing. J MedVirol. 2014; 86:1937-43.

[28] Ye J, Cheng X, Chen X, Ye F, Lu W, Xie X. Prevalence and risk profile of cervical Human papillomavirus infection in Zhejiang Province, southeast China: a population-based study. Virol J. 2010; 7:66.

[29] Dickson EL, Vogel RI, Bliss RL, Downs LS, Jr. Multiple-type human papillomavirus (HPV) infections: a cross-sectional analysis of the prevalence of specific types in 309,000 women referred for HPV testing at the time of 
cervical cytology. International journal of gynecological cancer. Int J GynecolCancer. 2013; 23:1295-302.

[30] Saslow D,Solomon D, Lawson HW, et al. American Cancer Society, American Society for Colposcopy and Cervical Pathology, and American Society for Clinical Pathology Screening Guidelines for the Prevention and Early Detection of Cervical Cancer. Am J Clin Pathol. 2012;137:516-542.

[31] Massad LS, Einstein MH, Huh WK, Katki HA, Kinney WK, Schiffman M. 2012 Updated ConsensusGuidelines for the Management of Abnormal Cervical Cancer Screening Tests and Cancer Precursors. Jof Low Genit Tract Dis. 2013;17(5):S1-S27. 